Poster Sessions

\title{
Nonmyeloablative Stem Cell Transplantation with CD8- Depleted or Unmanipulated Peripheral Blood Stem Cells: A Prospective Randomized Trial.
}

\author{
Evelyne Willems, MD ${ }^{1, *}$, Emilie Castermans ${ }^{1, *}$, Frédéric Baron, MD, PhD ${ }^{1}$, Etienne \\ Baudoux, MD ${ }^{1, *}$, Nadine Wanten ${ }^{1, *}$, Christophe Bonnet, MD ${ }^{1, *}$, Kaoutar Hafraoui, MD ${ }^{1, *}$, \\ Pascale Frère, $\mathrm{MD}^{1, *}$, Georges Fillet, $\mathrm{MD}, \mathrm{PhD}^{1}$ and Yves Beguin, $\mathrm{MD}, \mathrm{PhD}^{1}$ \\ ${ }^{1}$ Hematology, University of Liège, Liège, Belgium.
}

\begin{abstract}
Background: In a previous pilot study, we demonstrated that CD8-depletion of the graft apparently reduced the severity of AGvHD without impairing the GvL effect after peripheral blood stem cell (PBSC) transplantation with a nonmyeloablative conditioning (NMSCT).

Aim of the study: To evaluate the effect of CD8-depletion on graft rejection, AGvHD and CGvHD, and relapse.

Patients: 53 patients were randomised between CD8-depletion (group 1) $(n=25)$ and no manipulation (group 2) $(n=28)$. Two patients in the CD8 group were excluded for poor CD34+ cell count collected. Diagnoses were: AML ( $n=3)$, CML-AP $(n=2)$, MDS $(n=14), \operatorname{MPD}(n=3)$, CLL $(n=5)$, NHL $(n=14)$, MM $(n=8)$ and RCC $(n=2)$. Median age was 57 (range 36-69) yrs. After conditioning with 2 Gy TBI with $(n=39)$ or without $(n=12)$ fludarabine, patients received PBSC from family $(n=21)$ or unrelated $(n=30)$ HLA-matched donors. CD8-depletion was carried out using the Eligix system and GvHD prophylaxis consisted in CyA and MMF.

Results: CD8 depletion removed $96 \%$ of CD8+ cells so that the number of CD8+ cells infused was 6.8 vs $136.8 \times 10^{8}$ cells $/ \mathrm{Kg}$ in group $2(\mathrm{p}<0.0001)$. AGvHD of any grade was observed in 13 (56\%) patients in group 1 and $17(61 \%)$ in group $2(\mathrm{NS})$; it was grade $3-4$ in $1(4 \%)$ and $5(18 \%)$ patients in groups 1 and 2, respectively (NS). Limited and extensive CGvHD developed in 3 and 1 patients in group 1 and in 7 and 2 patients in group 2, respectively (NS). Nine patients in group 1 and 12 in group 2 received unmanipulated DLI for poor chimerism or disease progression. Eight ( 3 initial and 5 late) graft failures were observed in group 1 and one (late) in group 2. Full donor chimerism was achieved in $57 \%$ (group 1) and 50\% (group 2) at day 100, and in $73 \%$ (group 1) and 59\% (group 2) (NS) at 1 yr. The 2-yr OS and PFS rates are 55\% and $43 \%$ in group 1 vs $59 \%$ and $46 \%$ in group 2, respectively (NS). Four (17\%) patients died of their disease in group 1 vs $3(11 \%)$ in group 2 (NS). Two patients died of severe AGvHD in group 2 vs none in group 1.
\end{abstract}

Conclusion: In vitro CD8-depletion results in higher rates of graft failure after NMSCT. The incidence of acute and chronic GvHD is not reduced but there is a trend towards reduced severity of AGvHD. Relapse and survival rates are not changed by this strategy. 